\title{
Reflexiones en torno a los riesgos y peligros para los varones en Sonora
}

\author{
José Eduardo Calvario Parra*
}

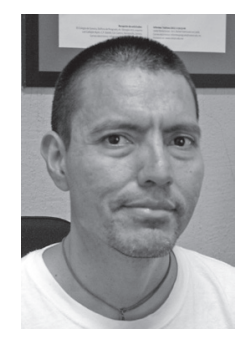

$M$ e he planteado un par de ideas e interrogantes con el fin de compartirles sobre el tema de la charla: "Los hombres en Sonora y los posibles cambios". En primer lugar, una pregunta orientará mis comentarios ¿en qué forma las transformaciones en las identidades masculinas en Sonora tienen que ver, o están relacionadas con el contexto de precariedad, vulnerabilidad y condiciones sociales y medioambientales adversas? ¿Qué hay de cierto en la afirmación de que existe una diferenciación en la exposición a amenazas y riesgos de todo tipo entre varones y mujeres? Y si es así, ¿es una fuente que alimenta las identidades de género?

Sonora no está sustraída a la inercia planetaria de la globalización, junto a ello, surgen nuevos riesgos y se reconfiguran viejos peligros. Las transformaciones de las relaciones de género en Sonora se imbrican con los procesos macrosociales como el mercado de trabajo, el impacto de las políticas públicas de salud y educación, la capacidad de movilidad social de distintas clases sociales; dichas imbricaciones están cargadas de peligros y riesgos. Para tratar de clarificar lo anterior, podríamos plantear dos ámbitos en los cuales se puedan identificar dichos peligros y riesgos con relación a las identidades de género:

I. En Sonora, la industria maquiladora y de servicio, junto con la industria agroexportadora, dan cuenta de un perfil de sus trabajadores/as que cada día se precariza más.

\footnotetext{
* Licenciado en Sociología por la Universidad de Sonora, Maestro en Ciencias Sociales con especialidad en salud por el Colegio de Sonora, Doctor en Ciencias Sociales por el Colegio de México. Profesorinvestigador Cátedras Conacyt en El Colegio Sonora, Profesor de asignatura en la Universidad de Sonora. joseduardo_calv@yahoo.com.mx
}

No creo que los varones y mujeres de los distintos puntos geográficos de México vivan de manera muy diferente dicha precarización, sin embargo, es posible que las cosmovisiones socioculturales de las regiones de Sonora hagan que las experiencias de las personas tengan matices particulares. Un ejidatario del valle del mayo, un obrero maquilador de Nogales, una ama de casa de Suaqui Grande, una empleada de Hermosillo, o un jornalero de la Costa de Hermosillo, comparten ciertos desafíos que les plantea su desempeño laboral y contexto particular. El ejidatario, el/la obrero/a, la empleada, la ama de casa y el jornalero/a tienen la impostergable necesidad de obtener un ingreso.

La obligatoriedad de proveer o "ser proveída" tiene variados matices según si se es varón, mujer u otra categoría para la distinción de género. Una constante que recorre sus experiencias biográficas tiene que ver con sus condicionantes de género. La experiencia de la precarización laboral se vive en torno al riesgo de desempleo, a la exclusión en los contornos de la economía informal. El imperativo de proveer al grupo doméstico es asumido por varones y mujeres; la diferencia estriba en que para los primeros, pesan en sus hombros doble presión: la cultural y la material. En las mujeres la necesidad económica está por encima de la obligatoriedad cultural.

Las ocupaciones están preñadas de género y por lo tanto, también la precarización. En la reconfiguración de la división sexual del trabajo, la distribución de los peligros y riesgos impacta en los géneros. Sin embargo, los efectos y las cargas de dichas distribuciones son distintos. Los mineros de Cananea, los tractoristas de la Costa de Hermosillo o los vaqueros de los pueblos de la sierra, seguramente viven la precariedad y distribución del riesgo de manera muy distinta a las cocineras y hosteleras que brindan asistencia a los mineros, tractoristas o vaqueros de esas misma regiones. 
Un indicador para entender la dimensión de las diferencias entre varones y mujeres, es la incapacidad laboral permanente en el IMSS; por ejemplo, para Sonora en el año 2008 resultó de 594 para los primeros y 150 para las mujeres. Las defunciones por riesgos de trabajo resultaron de 43 en los varones y solo un caso en las mujeres.

En la mayoría de las ocupaciones, los varones lideran los accidentes de trabajo, pero hay excepciones como en el de las ocupaciones de limpieza y cocina o están parejos como en las relacionadas con el comercio (ventas y servicio).

Lo anterior, ¿qué nos dice? Nos dice poco o nada sobre las transformaciones de las relaciones de género y su relación con los riesgos; sin embargo, nos permite constatar que la distribución de los efectos de los riesgos laborales es diferencial según género y ocupación.

II. Los cambios sociales son también cambios en las dinámicas de género; hay varios puntos en los cuales se puede rastrear las transformaciones. En América Latina y Sonora no es la excepción, se experimenta una disminución o estabilización de los matrimonios y aumento de los divorcios; aumenta también de manera sostenida el número de mujeres, jefes de hogar, en detrimento de los hogares con jefatura masculina. La masiva incorporación de las mujeres al mercado de trabajo trastoca el orden de género tradicional o pacto de género como lo refiere José Olavarría, sociólogo chileno especialista en el tema. Este conjunto de tendencias contribuye a presentar un nuevo rostro de la familia, por lo menos es su acepción tradicional y con ello, nuevas negociaciones en la distribución de las tareas y responsabilidades entre los géneros.

En Sonora, como en otros lugares, las tendencias no son homogéneas y por lo tanto, los cambios tampoco lo son. Es interesante observar un periodo de tiempo en lo que respecta a los matrimonios y divorcios. Tomando como referencia 1994 hasta la fecha, si bien la tendencia estatal es la estabilización del matrimonio y el aumento de los divorcios, esto no es así para un conjunto de municipios serreños. Existe un ligero aumento de los matrimonios y en algunos casos, muy pocos o ningún divorcio. Independientemente de las expresiones de las relaciones de conyugalidad informales, por ejemplo, la llamada unión libre, lo que es un hecho es la inestabilidad de los tipos de lazos, incluso asumen distintos rostros según región, clase social, edad, etnia. Es posible que en ciertas zonas, como por ejemplo en la mencionada sierra, se den expresiones de estructuras patriarcales que facilitan que los varones se resistan a participar en actividades domésticas 0 permitan que sus parejas laboren y a la vez, sean cariñosos, amorosos.

En términos generales, especialistas hablan de una crisis del orden de género, no estoy muy convencido que esto sea así para el caso de Sonora, o por lo menos que sea muy nítido, pero sin duda, los factores exógenos como la globalización y la precarización del empleo son unas fuentes que incentivan el cambio en las relaciones de género.

¿En qué forma los varones han cambiado ante dichos escenarios? ¿Los riesgos y peligros también son revalorados y resignificados? Es posible que varones de clase media,

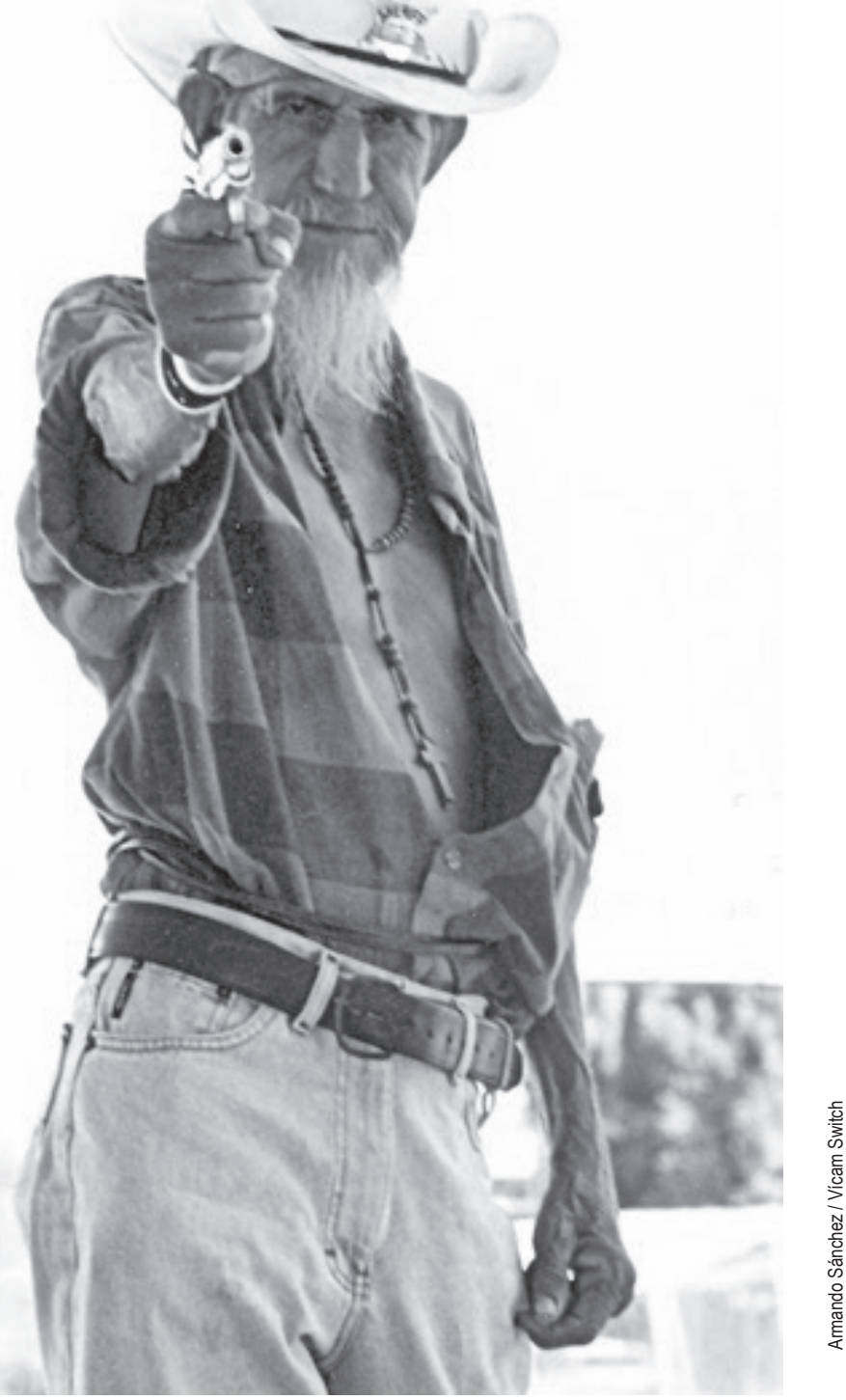

Brígido Jara, el Naylon. Personaje estrafalario, ícono del lugar, El Naylon fue siempre el eterno sheriff del pueblo. Los recién llegados (generalmente profesores provenientes de tierras lejanas) entraban en pánico cuando El Naylon les decía: "Hey, forastero", al mismo tiempo que su dedo índice simulaba un cuchillo sobre el cuello (A. Valenzuela).

educada y citadina (Obregón, Hermosillo, Nogales, San Luis Río Colorado, etc.), tengan prácticas que resignifiquen el modelo tradicional masculino (único proveedor, conquistador, valiente, autoritario, aguantador); pero también se puede registrar en medios rurales; la diferencia estiba en la intensidad y frecuencia de dichos cambios.

Es posible también, que tanto en las relaciones afectivas, amorosas, íntimas, se estén transformando. Los riesgos del modelo económico, la proliferación o institucionalización de la violencia y las desigualdades de género aún persistentes, hacen que los individuos, tanto en la vida privada como en la pública, se vean obligados a sortear dichos peligros con relación directa a las identidades de género. En el caso de los varones, la experiencia puede resultar amarga y angustiante. El desafío que plantean dichos escenarios no le corresponde solamente a uno de los géneros, sino también a las mujeres, en el ánimo de ir construyendo sociedades más igualitarias, menos violentas, con una garantía mínima de bienestar colectivo. 